\title{
Propuesta de un sistema de gestión integrado para fondos documentales patrimoniales familiares
}

\author{
Integrated heritage management systems for family holdings: a proposal
}

\author{
Camino SÁNCHEZ OLIVEIRA \\ Universidad de Zaragoza, Departamento de Ciencias de la Documentación e Historia de la Ciencia, \\ Pedro Cerbuna 12; 50009, Zaragoza, camsanch@unizar.es
}

\begin{abstract}
Resumen
Se define un sistema de gestión para lo que se ha denominado como un Fondo Documental Patrimonial Familiar (FDPF). Se reflexiona sobre los elementos que lo fundamentan y componen: un fondo bibliográfico (una biblioteca), un fondo archivístico (un archivo familiar) y, con bastante frecuencia, una colección de objetos materiales relacionados con las actividades de los generadores del FDPF. Se concluye que dicho sistema de gestión conjunto debe definirse en base a su conceptualización patrimonial, a su procesamiento documental y a su funcionalidad, y debe atender al tipo de centro a cargo de dicho patrimonio.
\end{abstract}

Palabras clave: Patrimonio documental. Patrimonio bibliográfico. Patrimonio cultural. Fondo documental patrimonial familiar. Sistema de gestión. Centro de información patrimonial. Archivos familiares. Bibliotecas familiares.

\section{Definición de Fondo Documental Patrimonial Familiar (FDPF): entre el patrimonio cultural y el patrimonio documental y bibliográfico}

Son muchos los estudios existentes sobre la definición y conceptualización que ha ido desarrollándose y evolucionando en torno al patrimonio cultural, histórico, documental y bibliográfico partiendo de la normativa legal, con el objeto de precisar qué se entiende por patrimonio y concretar las medidas que se han de adoptar para su conservación, protección y difusión. Desde los orígenes legislativos se hace una distinción entre $\mathrm{Pa}$ trimonio Documental y Bibliográfico, atendiendo al tipo de bienes, en este caso documentos, que los integran así como a su antigüedad y a las instituciones que los albergan y la titularidad de las mismas ya sean personas o entidades, físicas o jurídicas, públicas o privadas.

Este artículo propone acercarse al concepto de patrimonio documental y bibliográfico entendido

\begin{abstract}
A management system is defined for what has been coined as a Family Document Heritage Holding $(\mathrm{FDHH})$. The elements that underlie and compose it are discussed: a bibliographic collection (a library), an archival holding (a family archive) and, quite frequently, a collection of material objects related to the activities of the FDHH generators. It is concluded that this integrated management system must be defined on its heritage accrual processes and history, its document processing and its functionality, and must address the type of center custoding it.
\end{abstract}

Keywords: Archival heritage. Bibliographic heritage. Cultural heritage. Family heritage documentary holdings. Management system. Heritage information center. Family archives. Family libraries.

como "las expresiones artísticas, históricas, culturales, folklóricas, educativas, intelectuales, científicas, entre otras, que han sido producidas para ser testigo fiel del desarrollo de las sociedades; y que han sido objetivadas en manuscritos, impresos, medios audiovisuales, documentos electrónicos, etc., cuya finalidad es almacenar, transmitir, preservar, conservar, comunicar y difundir la suma de conocimientos que contienen" (Palma, 2013, 34). Es decir, se pretende ofrecer un concepto aglutinador, definiendo conjuntamente el patrimonio existente en bibliotecas y archivos como patrimonio cultural, denominado como objeto documental patrimonial, de tal manera que se reúnan el patrimonio bibliográfico y el archivístico, contemplando así una acepción de conjunto que ayude a reconocer la diversidad de documentos que puede abarcar el documento patrimonial o patrimonio documental, dicho en su sentido más amplio.

Del mismo modo, nos referimos al concepto de "fondo antiguo", entendido como un conjunto de 
objetos bibliográficos y documentales que atesora la riqueza, la esencia y el espíritu de la cultura, cuyos caracteres esenciales son el valor histórico, el estético y el valor del conocimiento (Mora, 2003, 202). Acudiendo a la acepción fundamental y vertebradora de "fondo", que se define como un "conjunto documental que llega hasta el presente y se caracteriza por poseer un origen común que lo dota de sentido, por lo que debe mantenerse reunido" (Pedraza, 2014, 46).

De esta manera, cuando hablamos de tratar un FDPF, nos hallamos, de manera unísona y cohesionada, ante lo que se puede denominar un "fondo de fondos": una biblioteca (un fondo bibliográfico) y un archivo (un fondo documental), que bien podría pasar a denominarse un fondo de objetos documentales patrimoniales. La biblioteca, en estos casos, suele ser el reflejo del status cultural, económico y social de alguno de sus miembros destacados o del conjunto de una generación. Y el archivo familiar, el resultado de las actividades, tanto personales como públicas en la administración de sus bienes y en el ejercicio de sus funciones, constituyendo el seguro documental de lo que la familia tiene que defender y justificar: propiedades, derechos, jurisdicción, honores y privilegios que se quieren perpetuar. No podemos olvidar que estos fondos se acompañan, a menudo, de un sinfín de objetos materiales (placas de reconocimiento y condecoraciones, uniformes, objetos personales...) relacionados con esas mismas actividades y sus generadores.

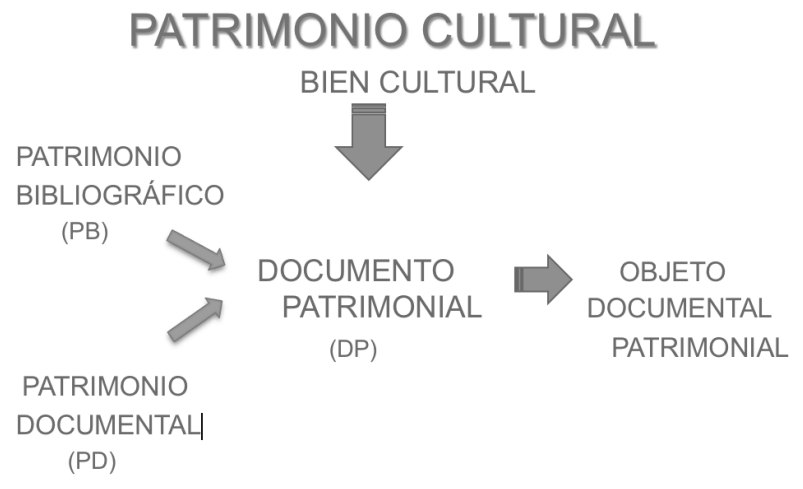

Figura 1. Definición de un FDPF

La familia es el sujeto generador o productor que da sentido, cohesión, forma y determinación a un FDPF. Pues este fondo es el reflejo de las diversas acciones, actividades e intereses de sus diferentes miembros a lo largo de varias generaciones, tanto de manera individual como, sobre todo, colectiva. Nos referimos al concepto de familia extensa, no solo nuclear, como eje vertebrador de la sociedad, la política y la economía relativas a entornos o esferas de influencia de carácter principalmente local, que hasta finales del siglo XIX mantuvieron toda su influencia y razón de ser.

\section{Un Sistema de gestión integrador para los FDPF}

\subsection{Entre la gestión patrimonial} y la gestión documental

Es preciso proponer un sistema de gestión para la documentación patrimonial familiar, que se derive por igual tanto de sus funcionalidades como patrimonio cultural, como por la esencia misma del tipo de documento y el tratamiento aplicado para procesar cada tipología documental. De tal manera que los retos de la práctica patrimonial se integren con los de la práctica documental, gestionando el legado científica y adecuadamente dentro de su variada tipología, aunando las funciones tradicionales de investigación y preservación, hacia una difusión más mayoritaria, incluso -si cabe- como agente social.

Dos son las formas de gestión que se ven conjugadas en este sistema de gestión integrador: la gestión del patrimonio como bien cultural y la gestión documental. La gestión patrimonial viene determinada de manera inequívoca en las acciones que se deben llevar a cabo para cumplir con las funcionalidades que tiene la documentación patrimonial, y que aparecen claramente definidas en la Ley 16/1985 (art. 62) en lo referente a los bienes documentales y bibliográficos: conservarse y difundirse. Estas actuaciones forman una cadena que se basa en la lógica y las metodologías científicas: el patrimonio se investiga, se protege, se conserva y se restaura para finalmente difundirse (Tugores y Planas, 2006, 80). Se trata de gestionar para conservar y difundir (a la sociedad-comunidad interesada). Y la gestión documental define el aprovechamiento de los recursos informacionales para promover acciones que procuren un aprovechamiento máximo del uso y procesamiento documental para beneficio del funcionamiento del propio centro de información, en correspondencia con su misión, objetivos y operaciones. Se trata de gestionar para sistematizar o seguir gestionando, además de para conservar y difundir.

Los dos sistemas confluyen y se complementan. Son los propios fondos documentales patrimoniales los recursos principales que definen un sistema de gestión integrado al establecer las acciones que van destinadas a su tratamiento para la consecución tanto de su fin último-patrimonial: la conservación y difusión; como el de sus fines 
intermedios- organizativos-documentales: establecer y definir el funcionamiento de la institución.

\section{FONDO DOCUMENTAL PATRIMONIAL FAMILIAR (OBJETO DOCUMENTAL-BIEN CULTURAL)}

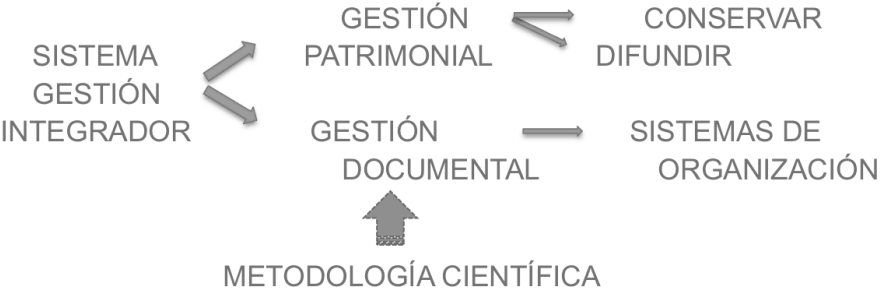

Figura 2. Sistema de gestión integrador

Se fundamentan además en un mismo principio: basarse en metodologías científicas, muy arraigado en la ciencia archivística, dicho sea de paso. En materia de patrimonio, investigar tiene como objeto decodificar toda la información de la que son portadores los objetos patrimoniales, tanto como piezas individuales como en su conjunto, para incrementar el conocimiento sobre ellos y las sociedades, en este caso circunscrito al entorno de la familia, definiendo así a la propia institución que los alberga y gestiona. La investigación es el hilo conductor que debe mantener una relación estructural con y para el centro, asociada a su misión principal: hacerla accesible para la investigación. Los valores patrimoniales solo se desvelan mediante su investigación, por ello hay que señalar también que la información sobre el patrimonio cultural no se reduce únicamente a la que generan las propias instituciones (en forma de inventarios, catálogos, etc.), sino que se completa con los trabajos de investigación. Se cierra así un ciclo que comienza y acaba con la investigación.

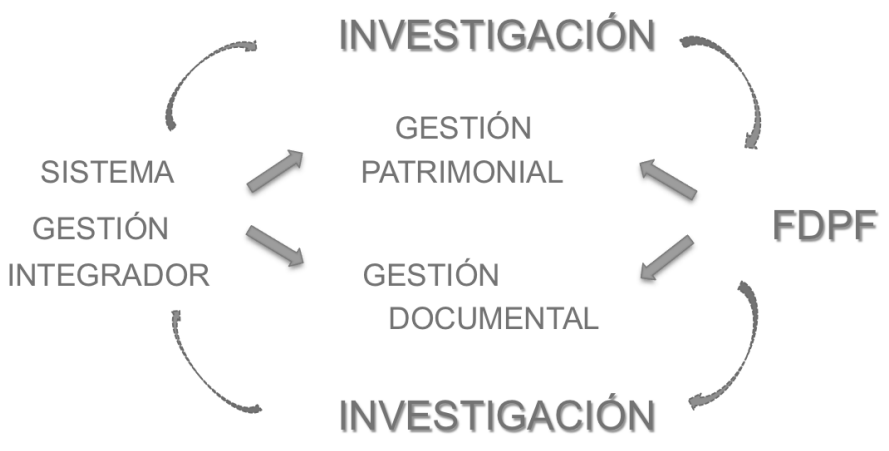

Figura 3. Sistema de gestión integrador
2.2. Las bibliotecas y los archivos como sistemas de información diferenciados

Nos interesa descender ahora en el nivel de gestión documental, donde la investigación es el proceso esencial en cualquier intervención que ha de servir de fundamento a los niveles restantes. La Ley 16/1985 (art. 59) menciona de manera más concreta las acciones que se deben dar a los fondos patrimoniales, que no son otra cosa que las funciones que el centro debe desarrollar para procesarlos. Establece una cadena sucesiva de etapas o tareas, propias del proceso documental, y las distingue según la institución responsable: los archivos reúnen, conservan, ordenan y difunden. $Y$ las bibliotecas conservan, reúnen, seleccionan, inventarían, catalogan, clasifican y difunden. Ambas confluyen en el inicio y en el final del proceso, pero es significativo marcar la diferencia de delimitación de funciones organizativas que se establecen para cada tipología documental e institución gestora. Vinculado a este proceso o cadena de tareas, se hallan las herramientas o instrumentos que se elaboran para la organización y descripción documental: guías, cuadros de clasificación, inventarios, catálogos, etc. Todas ellas, sin duda, deben adaptarse a cada tipología y fondo específico.

\section{FONDO DOCUMENTAL PATRIMONIAL FAMILIAR OBJETO DOCUMENTAL

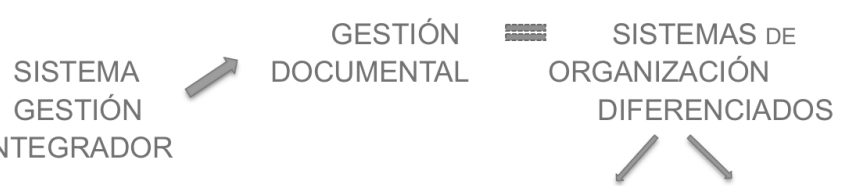 \\ ARCHIVOS BIBLIOTECAS}

Figura 4. Sistema de gestión integrador

Analizando más detalladamente el proceso de organización documental y las diferentes tareas que lo integran, se debe separar el fondo bibliográfico, por un lado, y el archivístico, por otro, teniendo que hablar de las bibliotecas y los archivos como sistemas de información diferenciados.

Según las teorías archivísticas, el archivo requiere una organización científica, programada y jerarquizada. Este tipo de organización se debe a la singularidad del documento de archivo como parte indivisible de un conjunto superior en que se halla integrado. Se establece un orden indispensable de realización de las tareas: encabeza la clasificación, que es la función básica e imprescindible del tratamiento archivístico que debe atender a los principios básicos de 
procedencia y respeto al orden original. Esta fase determina y potencia las cualidades informativas de un archivo, y hace que como sistema tenga un modelo muy concreto de representación y organización del conocimiento, que se refleja en su herramienta fundamental: el cuadro de clasificación, donde los documentos se encuadran en un conjunto orgánico del que forman parte y en el que adquieren todo su valor informativo. Le sigue la tarea de la ordenación y, de manera diferenciada, continúa la descripción, que se concibe como una forma de planificar la información acerca de los fondos y se materializa mediante la aplicación de los instrumentos de descripción más adecuados, según los criterios de especificidad, prioridad o preferencia: guías, inventarios y catálogos.

\section{ARCHIVOS \\ SISTEMA DE INFORMACIÓN}

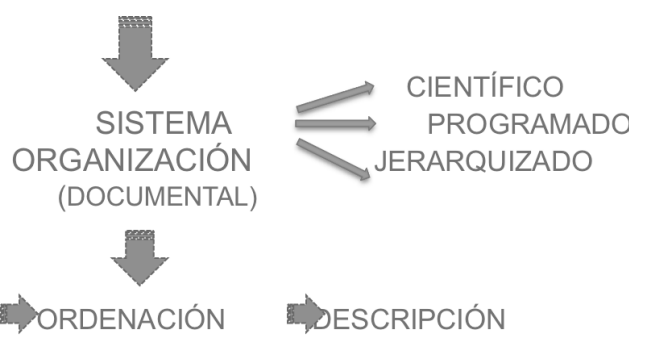

Figura 5. Archivos: sistema de información

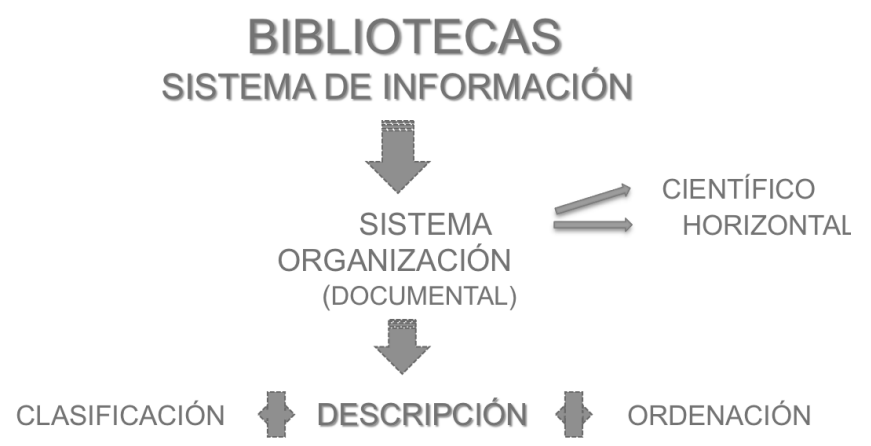

Figura 6. Bibliotecas: sistema de información

En el caso de las bibliotecas, la organización es lineal, se establece documento a documento. La tarea de clasificación como organización conceptual del conocimiento de base científica, se realiza mediante clasificaciones bibliográficas o lenguajes documentales controlados y no controlados. Paralelamente, pero no secuencialmente, está la tarea de la descripción normalizada, que se convierte en la base informativa por excelencia en las bibliotecas, como forma principal de recuperación de la información mediante los catálogos. Ambas fases determinan la ordenación de los libros. En este sistema organizativo el acercamiento a la pieza individual se impone, la mayoría de las veces sobre el conjunto, pero no olvidemos que en este caso estamos hablando de libros antiguos, de fondo antiguo, donde la visión de conjunto como colección es parte indispensable de la potencialidad informativa de un fondo bibliográfico patrimonial.

\subsection{Centros de gestión: entre los Archivos Históricos y las Bibliotecas Patrimoniales}

La Ley 16/1985 del Patrimonio Histórico Español (art. 59) especifica que son instituciones culturales los centros que deben hacerse cargo y albergar tales fondos, y diferencia igualmente entre bibliotecas y archivos, según se trate de patrimonio bibliográfico o documental. Lo habitual es disgregar y desvincular el fondo bibliográfico del documental en instituciones con formas de gestión diferenciadas. En general, legislativa y administrativamente, se habla siempre de instituciones de titularidad pública. No obstante, se deja cabida al carácter privado - como organismos generadores más que gestores- de tal manera que los propietarios por ellos mismos o constituyéndose en fundaciones custodien la documentación. Se suele ofrecer como alternativa a la gestión privada la cesión al Estado por medio de las distintas vías existentes: donación, venta, depósito o cesión, entre otras.

Desde la dualidad antes comentada se deben señalar los dos tipos de centros existentes en la actualidad para albergar y gestionar este, podemos decir, conjunto de fondos: por un lado están los Archivos Históricos, como aquellos que albergan documentos del final del "ciclo vital" del documento archivístico sin función administrativa, gestionados y conservados para su preservación y difusión. Dentro del sistema archivístico, son los archivos que siempre han tenido como base la gestión documental y la concepción de la ciencia archivística por sus funciones histórico-culturales y de investigación. Para el caso de los archivos familiares existen varios centros de referencia sobre el tratamiento y custodia de dichos archivos, que se diferencian según la región o Comunidad Autónoma y el modelo social-patrimonial-territorial que allí predomina. Por ejemplo para los de carácter nobiliario, la Sección Nobleza del Archivo Histórico Nacional o el Arxiu Nacional de Catalunya, por citar algunos.

Y por el otro lado, están las Bibliotecas Patrimoniales, en su mayoría públicas y universitarias, que a veces constituyen una sección de la misma $y$ en otras se constituyen en una biblioteca con objetivos y sentido por ella misma. Es interesante 
acercarse a este tipo de bibliotecas desde la visión más actual que las define como centros de información, que se diferencian de otras instituciones por el tipo de documentación que albergan (fondos bibliográficos antiguos) y por el tratamiento documental-funcional específico que dan a dichos fondos. En ellos los documentos que se custodian son tratados como objetos documentales patrimoniales que tanto pueden ser estudiados como unidades bibliográficas como en conjunto. Esto es, como parte del fondo o colección de la que provienen, que siempre debe ser identificado y mantenido reunido. Dicho de otro modo, la definición de biblioteca patrimonial como centro de información deriva de las características propias de la documentación que alberga y maneja, a la que adapta toda su estructura organizativa y de gestión, con el fin fundamental de preservarla y hacerla accesible para la investigación, siempre desde su dos enfoques posibles: individual y de conjunto.

El planteamiento de poder establecer un sistema y una institución común no es un debate reciente: en el Congreso Internacional de Archivos de 1988 de la IFLA ya se planteó la cuestión sobre la integración o la creación de instituciones especificas, y se abogó por la unidad de sistemas de información, pronunciándose a favor de la integración de archivos, bibliotecas y centros de documentación. Eso sí, se defendía una integración con diferenciación: una ubicación en un mismo local, donde se pudiese distinguir claramente la separación física independiente de cada tipología documental, con su tratamiento orgánicodescriptivo diferente, aunque la información que se obtiene de cada uno sea complementaria (Heredia Herrera, 1995, 170).

\section{3. ¿Podemos hablar entonces de pacto patrimonial? Dificultades y posibilidades de una integración}

El concepto de patrimonio iguala las diferencias existentes entre las diferentes tipologías documentales, los centros que los tratan y sus sistemas de información, organización y tratamiento documental, pues, no olvidemos, dentro de las bibliotecas, hablamos de Bibliotecas Patrimoniales, y dentro de archivos, de Archivos Históricos. Ambas realidades pueden confluir en un sistema de gestión integrado por varios motivos:

Guardan un principio común y fundamental ya comentado: el uso de una metodología científica, con la que se realiza un procesamiento documental exhaustivo y específico, ya sea sobre un documento bibliográfico o uno archivístico.

Los recursos informacionales son documentos primarios y/o únicos: el documento de archivo lo es por su propia naturaleza, pero el libro antiguo posee también ese carácter de unicum, porque en este caso no solo forma parte como un impreso de una tirada determinada de una edición concreta, sino que cada volumen por sus características propias de ejemplar, es reflejo de un devenir y una singularidad por la que debe ser tratado y considerado como un documento sin igual. Por eso podemos hablar de manera unificada de objetos documentales patrimoniales.

La potencialidad informativa, su máximo valor informativo, se extrae junto a su valor patrimonial, que - recordemos - solo se desvela mediante la investigación y desde su tratamiento de conjunto. El concepto de fondo es la acepción indiscutible, de tal manera que el objeto patrimonial documental debe ser estudiado conjunta e indisolublemente para poder ser procesado adecuadamente. En este caso es cuando el concepto de fondo frente al de colección -como conjunto documental creado artificialmente con unos fines específicos-cobra mayor relevancia y sentido. Todo ello, sin entrar en profundidad en ese conjunto de objetos materiales sin determinar que no suelen tener cabida ni en archivos ni en bibliotecas, pero que adquieren pleno sentido dentro de un FDPF.

Ambos son además sistemas de información limitados por las medidas cautelares de conservación y preservación de originales, que deben hacer compatibles con su leitmotiv: la difusión. Con la difusión se cumple la función social y obligada del patrimonio, pero también se asegura su conservación: aquello que es conocido, comprendido y valorado difícilmente será destruido. Hay que procurar su acceso, y esa condición sine qua non no está libre de un complejo debate, que se dirime siempre entre la pertinencia de permitir el acceso al documento o a la información que contienen, así como las vías más adecuadas para ello. Deliberación a la que deben sumarse las nuevas necesidades de uso de esa información o documentos de cara a una difusión no solo especializada, abriendo la posibilidad de hacerla compatible y extensible a una de carácter más divulgativo.

Se puede establecer una sinergia entre ambas realidades institucionales y ofrecer una propuesta para la definición de un Centro de Información Patrimonial (CIP) ligado al sistema de gestión integrador que se propone y a los elementos que lo fundamentan. Hablamos entonces de una institución que debe estar estructurada por el método y las prácticas científicas. Como centro de información, su gestión deriva de las características propias de la documentación que alberga y maneja, a la que adapta toda su estructura organizativa y de gestión, con el fin 
fundamental de preservarla y hacerla -en primera instancia- accesible para la investigación, siempre desde sus dos enfoques posibles: individual y de conjunto. Un CIP ligado al sistema de gestión integrador propuesto, como centro especializado en torno al fondo que alberga, podría superar la dualidad institucional existente, y aproximarse a unas prácticas de difusión, más como centro cultural, llegando a superar la función tradicional de difusión para los investigadores y alcanzar una más mayoritaria, incluso -si cabecomo agente social, lo que conllevaría a acercarse, sin duda, a prácticas museísticas.

\section{SISTEMA DE GESTIÓN INTEGRADOR}

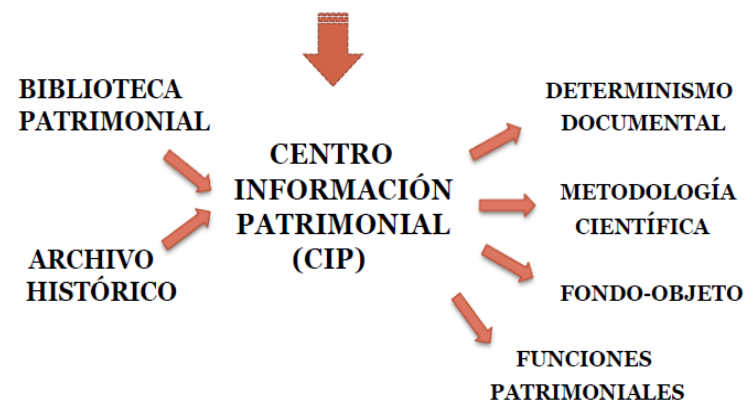

Figura 7. Elementos de un centro de información patrimonial

La clave se encuentra en la complementariedad de la información que contienen los fondos y en cómo gestionarla para que esa información sea procesada de manera ágil, pertinente, eficaz y así poder ser compartida y aplicable en las diferentes fases de tratamiento del Fondo Documental Patrimonial Familiar que se tiene entre manos. En este sentido, destacamos la fase o tarea documental, de la identificación, que es la primerísima fase de la organización, ya sea archivística o bibliográfica, que en el caso de los FDPF es definitoria y transversal a todo el proceso organizativo, no solo en esa fase teóricamente inicial. Dicha identificación de los objetos documentales antiguos es fundamental y no siempre es tarea fácil. Se requieren unos conocimientos muy amplios y variados, que oscilan desde los lingüísticos y paleográficos hasta los específicos de cada disciplina - de las muchas que abarcan este tipo de objetos patrimoniales-, que deben aplicarse sobre un método de identificación científico fiable e incuestionable: un error en esta fase, desencadenaría un cúmulo de errores catastróficos. Es en esta fase del tratamiento documental, donde el concepto de FDPF cobra sentido en sí mismo. Su análisis en origen nunca debe ser sesgado ni indiferente. A menudo, los documentos de archivo nos remiten a los libros y a la biblioteca del fondo, ya sea por medio de inventarios, libros de cuentas, de la correspondencia, etc.; y en ocasiones, los libros nos hablan, y hasta traen consigo documentos de archivo: testigos dejados en el interior de los libros en forma de cartas personales, notas de cuentas, naipes, documentos académicos o jurídicos del propietario del libro, etc. Y solo si se tiene mucha suerte, se encuentran o llegan hasta el presente de manera conjunta, dando una información identificativa privilegiada y una perspectiva histórica del devenir de una familia con el paso del tiempo: ¿tiene sentido separarlos?

Consecuentemente a la identificación, sigue la fase de la selección, que obedece y bebe de los criterios y la metodología establecida para delimitar el contenido del fondo o -lo que es más peligroso- la determinación certera de la eliminación o expurgo de parte de ese fondo.

Este carácter indisoluble de la información y la necesidad de mantenerlo lo volvemos a encontrar a la hora de elaborar las herramientas o instrumentos de organización y descripción antes aludidos, tan necesarios para la difusión documental e informacional: en las guías, cuadros de clasificación, inventarios, catálogos, etc., en los que de alguna manera hay que vincular y representar esa conexión entre fondos. Es un asunto que ya está presente en los debates sobre las prácticas de ambas disciplinas de las que partimos. Por ejemplo, la ciencia archivística, en la elaboración de los cuadros de clasificación deja cabida a indicar como parte del fondo propiamente documental, en un genérico pero siempre presente apartado de "Colecciones", esa otra documentación hallada en los archivos, los "libros, las publicaciones periódicas y la propaganda" (Gifre, P., Matas, J., Soler, Santi, 2002, 34-35) como documentación impresa no propia de los archivos, pero que refleja los intereses de una familia, sus actividades - su mundo, en definitivay por ello deben incluirse y considerarse como parte del fondo. Es más, se afirma que es tarea del centro que los agrupa y diferencia entre biblioteca, hemeroteca y archivo, dejar testimonio de su origen común, incluyendo siempre una relación entre esos materiales complementarios en la descripción de cada fondo.

Este vínculo informativo e informacional hay que hacerlo llegar al momento de la difusión y acceso a la información: lo usual es hacerlo por medio de las herramientas tradicionales de descripción antes comentadas, siendo en este ámbito donde el desarrollo de la tecnología informática amplía claramente las posibilidades.

El modelo que se propone de gestión conjunta de este tipo de FDPF se encuentra, en la práctica, ejemplificado más para colecciones que para fondos propiamente dichos (pudiéndose acuñar 
incluso el término de Colecciones Documentales Patrimoniales de manera diferenciada), donde, sí se localizan más opciones de gestión privada. Como ejemplo de ello mencionamos el caso del Archivo y la Biblioteca de Francisco Zabálburu en Madrid. Y sobre todo lo hallamos para casos concretos de fondos o colecciones de figuras o personajes individuales (no familiares) de reconocido prestigio social, político y económico (pudiendo del mismo modo hablar así de otro tipo de fondos o colecciones patrimoniales de carácter personal). De esta manera, se debe hacer mención explícita a los archivos personales. Un tipo de archivo - que pese al nombre acoge realidades documentales mucho más diversas- muy asociado y tratado por la bibliografía de manera similar a los archivos familiares, y para el que se debe considerar una gestión integradora y de conjunto - actualmente, además, existe un vivo debate institucional en torno a sus formas de trabajo y aplicaciones científico-documentales. Valga de ejemplo el escenario de tener que procesar documentalmente la biblioteca, la correspondencia y cualquier otro tipo de documento de carácter personal y/o profesional de un personaje de relevancia literaria o musical, y que confluyen de tal modo que no se puede explicar lo uno sin lo otro, pasando el conjunto - o fondo- a formar parte de una misma institución, generalmente una biblioteca patrimonial. Resaltamos para este tipo de archivos personales el papel que lleva a cabo la Biblioteca Nacional de España en su gestión y difusión o casos más circunscritos como el de la Biblioteca Regional de Madrid "Joaquín Leguina", y su labor de albergar fondos vinculados a personajes de relevancia del entorno madrileño. Como se ha visto, el punto de partida son marcos institucionales con unos sistemas de información adaptados principalmente -es verdad que según la institución en distinto grado- al tratamiento de la tipología documental de una de las dos ciencias: la archivística o la bibliotecológica. En donde con este tipo de fondos se tienen que enfrentar a la implementación (y resolución) de todas las cuestiones de gestión antes expuestas.

El devenir futuro - que ya es presente- de estos fondos pasa además por un proceso que parece, hoy en día, inevitable e incuestionable y que debe añadirse a todas esas tareas o fases del tratamiento documental: la digitalización, que se presenta generalmente como una solución no solo a la conservación sino también para establecer -en nuestro caso mantener- estos vínculos informacionales. La gestión digital aparece así con un necesario replanteamiento del concepto y modo de análisis, tanto teórico como metodológico, del patrimonio documental, como la clave de su supervivencia y quién sabe si en el futuro, -incluso-, de su justificación por su rentabilidad. Sin duda, es el reto que deben asumir los centros encargados de su gestión como bien cultural, que les lleva a acercarse a prácticas y concepciones museísticas, que eliminan fronteras y amplían posibilidades en cuanto a la forma de gestión y de comunicación e interacción social. La gestión digital abre, además, otra posibilidad: centros de información de gestión $-\mathrm{y}$ no depósito- que hagan de intermediarios entre las instituciones albergadoras y la comunidad a la que se dirigen. Un caso de interés en el que merecería la pena indagar más sobre su sistema de gestión e informacional es la propuesta que ofrece el centro responsable de la gestión del patrimonio cultural de la región aragonesa, eso sí, en el ámbito de "todo tipo de bienes (desde el patrimonio etnográfico a la cultura inmaterial) en estrecha vinculación con un espacio y una cultura determinados" (Bolea, Francisco; Diego, Jesús de, 2001, 118): el Centro de Información del Patrimonio Cultural Altoaragonés (CIPCA).

\section{A modo de conclusión}

Se ha presentado una propuesta de definición y conceptualización de un Fondo Documental Patrimonial Familiar (FDPF). Entre manos tenemos una variada tipología patrimonial documental, pero por encima de las peculiaridades de cada una, inherente a su propia naturaleza documental y organizativa, se pueden - se deben- hallar criterios de gestión conjuntos.

Del mismo modo, se ha tratado de determinar y reflexionar sobre los elementos que fundamentan un sistema de gestión integrador, patrimonial y documental, que se resumen en: determinismo documental (en su tratamiento y funcionalidad); metodología científica; en su análisis integrado como fondo y objeto documental patrimonia; $y$, por supuesto, de cara a sus dos funcionalidades patrimoniales principales: conservarse y difundirse.

En un FDPF, los fondos bibliográficos y archivísticos, deben ser diferenciados e integrados, pues están conectados por la propia documentación que albergan: por la información complementaria que contienen, y la forma y la ubicación en la que los encontramos en su génesis. El concepto de fondo es la acepción indiscutible, de tal manera que el objeto patrimonial documental sea estudiado individual, conjunta e indisolublemente para poder ser procesado de manera adecuada. Un sistema de gestión integrador como FDPF puede garantizar la perduración revivida del patrimonio y el desarrollo de sus funcionalidades tradicionales, incluso hallando nuevas, eso sí, siempre desde criterios científicos.

Otra cuestión es la selección del centro al que se deriven y en el que se gestionen los fondos. La 
gestión científica e integrada requiere recursos y personal especializado, que resultan costosos y de difícil viabilidad. Tradicionalmente la dualidad de gestión institucional y de carácter público se impone, frente a otras opciones mixtas o integradas y/o de carácter privado. Es muy difícil hacer compatible al generador o propietario original (privado-particular) con el gestor, no solo por medios y conocimientos, sino porque en los centros privados la Administración no puede imponer un modelo o criterios, solo legislar convenientemente en aras de conservar el patrimonio y proponer medidas.

Para finalizar, deberíamos plantearnos una pregunta ya latente: ¿Qué futuro tiene la gestión de la documentación patrimonial en la nueva concepción de representación del conocimiento, que se define como información electrónica, productiva y útil? Si esta premisa se impone, la gestión digital aparecerá en escena con un necesario replanteamiento funcional de los sistemas de gestión y del objeto documental patrimonial, pues... ¿Acabaremos en un futuro principalmente hablando de gestión de objetos digitales?

\section{Referencias}

Bolea, Francisco; Diego, Jesús de (2001). El Centro de Información del Patrimonio Cultural Altoaragonés: un modelo para el tratamiento y administración del patrimonio cultural mediante la gestión de sistemas de información integrada. // Scire. 7:2 (jul.-dic. 2001) 117-130.

Frías, José Antonio; Travieso, Críspulo (eds.) (2003). Tendencias de investigación en organización del conocimiento. Salamanca: Universidad de Salamanca, 2003.

García Aser, Rosario; Lafuente Urién, Aránzazu (2000). Archivos nobiliarios: cuadro de clasificación: sección nobleza del archivo histórico nacional. [Madrid]: Ministerio de Educación, Cultura y Deporte, Centro de Publicaciones, D.L. 2000

García Marco, Francisco Javier (1998). Organización del conocimiento y desarrollo de colecciones. // Carmen Negrete Gutiérrez (coord.). Primer seminario internacional sobre desarrollo de colecciones. México: Universidad Nacional Autónoma, 1998. 3-16.

Gifre, P., Matas, J., Soler, Santi, (2002). El arxius patrimonials, El arxius patrimonials, Girona, Associaciò d'historia rural de les comerques Gironines, 2002.

Heredia Herrera, Antonia (1995). Archivística general: teoría y práctica. Sevilla: Diputación de Sevilla. Publicaciones de la Diputacion Provincial de Sevilla, 1995. ( $7^{\mathrm{a}}$ ed.). 155170 .

Hernández Hernández, Francisca (1996). El patrimonio documental y bibliográfico. // Revista general de información y documentación. 6:1 (1996) 11-41.

Ley 16/1985, de 25 de junio, del Patrimonio Histórico Español (B.O.E. n. 155 de 29 de junio de 1985). <http://noticias.juridicas.com/base_datos/Admin/l16-1985.html> (201703).

Mora Alonso, Margarita (2003). El fondo antiguo como documento histórico: aproximación al siglo XVIII desde la Biblioteca Regional de Castilla-La Mancha. // Docampo Capilla, Javier (coord.). I Jornadas sobre Patrimonio Bibliográfico en Castilla-La Mancha: actas 12, 13 y 14 de noviembre de 2003, Toledo. Toledo: Junta de Comunidades de Castilla-La Mancha, 2003. 201-215.

Núñez Fernández, Eduardo (1999). Organización y gestión de archivos. Gijón: EdicionesTrea, 1999.

Pagarolas Sabaté, Laura (2007). Los archivos notariales, qué son y cómo se tratan. Gijón: Ediciones Trea, 2007.

Palma Peña, Juan Miguel (2013). Valores sociales y valores patrimoniales: elementos para determinar la significación del patrimonio documental // Biblioteca Universitaria: Revista de la Dirección de Bibliotecas de la UNAM. 16:1 (2013) 33-45

Pedraza Gracia, Manuel José (2014). Algunas reflexiones sobre bibliotecas históricas o patrimoniales: nuevo paradigma entre los centros y servicios de información // Investigación bibliotecológica.28:64 (2014) 33-50.

Ponjuán Dante, Gloria (2007). Gestión de la información: dimensiones e implementación para el éxito organizacional. Gijón: Ediciones Trea, 2007.

TugoresTruyol, Francesca; Planas Ferrer, Rosa (2006). Introducción al Patrimonio cultural. Gijón: EdicionesTrea, 2006.

Enviado: 2017-03-29. Segunda versión: 2018-01-25. Aceptado: 2018-06-21. 